Теорія Ймовір. та Матем. Статист. Вип. 80, 2009
Theor. Probability and Math. Statist.

No. 80, 2010, Pages 1-14

S 0094-9000(2010)00790-3

Article electronically published on August 18, 2010

\title{
EVALUATION OF BIAS IN HIGHER-ORDER SPECTRAL ESTIMATION
}

UDC 519.21

\author{
V. V. ANH, N. N. LEONENKO, AND L. M. SAKHNO
}

\begin{abstract}
This paper is concerned with the estimation of integral functionals of higher-order spectral densities for stationary random fields. It is shown that in some cases the problem of bias due to edge effects can be resolved by tapering.
\end{abstract}

\section{IntRoduCtion: THE PROBLEM AND BACKGROUND}

Let $X(t), t \in I$, be a real-valued measurable strictly stationary zero-mean random field, where $I$ is $\mathbb{R}^{d}$ or $\mathbb{Z}^{d}$ endowed with the measure $\nu(\cdot)$ which is the Lebesgue measure or the counting measure $(\nu(\{t\})=1)$, respectively. Suppose that the field $X(t)$ has spectral densities of order $k=2,3, \ldots$, that is, there exist complex-valued functions $f_{k}\left(\lambda_{1}, \ldots, \lambda_{k-1}\right) \in L_{1}\left(\mathbb{S}^{k-1}\right)$ such that the cumulant function of order $k$ is given by

$$
c_{k}\left(t_{1}, \ldots, t_{k-1}\right)=\int_{\mathbb{S}^{k-1}} f_{k}\left(\lambda_{1}, \ldots, \lambda_{k-1}\right) \exp \left\{i \sum_{j=1}^{k-1}\left(\lambda_{j}, t_{j}\right)\right\} d \lambda_{1} \cdots d \lambda_{k-1},
$$

where $\mathbb{S}=\mathbb{R}^{d}$ or $(-\pi, \pi]^{d}$ for the continuous-parameter or discrete-parameter cases, respectively. Here $\left(\lambda_{j}, t_{j}\right)$ is the inner product of the $d$-dimensional vectors $\lambda_{j}$ and $t_{j}$.

Let the field $X(t)$ be observed over the domain $D_{T}=[-T, T]^{d} \subset I$. We will consider the problem of estimation of integrals of cumulant spectra of orders $k=2,3, \ldots$ (spectral functionals)

$$
J_{k}\left(\varphi_{k}\right)=\int_{\mathbb{S}^{k-1}} \varphi_{k}(\lambda) f_{k}(\lambda) d \lambda
$$

for appropriate functions $\varphi_{k}(\lambda)$ with $\varphi_{k}(\lambda) f_{k}(\lambda) \in L_{1}\left(\mathbb{S}^{k-1}\right)$ based on the observations $X(t), t \in D_{T}$.

The functionals (2) can be used to represent some characteristics of stochastic processes and fields in a nonparametric setting. These functionals also appear in the parametric estimation in the spectral domain, e.g., when the minimum contrast (or quasilikelihood) estimators are studied. As examples, we mention estimators based on the Whittle functional and the functionals investigated in [20]. Estimators based on the Kullback-Leibler divergence considered in [1]-3] use information of the spectral densities not only of the second order but also of higher order. To study the properties

2000 Mathematics Subject Classification. Primary 62M15, 62M30.

Key words and phrases. Spectral estimation, bias, higher-order spectral densities, minimum contrast estimation.

Partly supported by the Australian Research Council grant DP0559807 and the Commission of the European Communities grant PIRSES-GA-2008-230804 within the programme "Marie Curie Actions". 
of minimum contrast estimators based on different contrast functionals (objective functions), one needs to know the limit theorems for the spectral functionals of the form (2) with particular weight functions $\varphi_{k}$. Functionals of the second order have been mostly used for these purposes and, therefore, have been extensively studied by now. But with the minimum contrast techniques based on higher-order spectral densities (as those elaborated in [1-3]), one needs the limit theorems for the functionals (2) with $k>2$. Besides, we should mention that the expressions for the variance matrices in the limiting normal distribution for minimum contrast estimators contain spectral functionals, and in order to apply asymptotic theory, these functionals have to be estimated. For example, the expression for the variance for the estimators based on information of the second order for non-Gaussian processes contains the integrals of the second- and fourth-order spectral densities. If the estimators based on higher-order information are used, then one needs to estimate various functionals of higher-order spectral densities; some of these functionals are of the form (2). More complicated nonlinear spectral functionals will be addressed elsewhere.

As one would immediately suggest, a natural way to construct the estimator for the functional (2) is to replace the spectral density $f_{k}(\lambda)$ in the integral (2) by a "good" estimator for it. In the estimation of spectral densities of order $k=2$ and higher orders, which have been described in nonparametric statistics, the periodograms of the second and higher orders and their modifications based on tapering (tapered periodograms), smoothing (kernel estimators), or tapering and shift-in-time methods have been extensively investigated. Periodograms constructed via the use of unbiased estimators for covariances and higher-order moments (or cumulants) with weighting and/or truncation have also been suggested.

The literature on estimating the spectral densities and asymptotic properties of the periodograms is rather voluminous. Some references relevant to our exposition are 4][10, [17, 21, 22.

We will study in the present paper the estimators for the functional $J_{k}\left(\varphi_{k}\right)$ based on tapered periodograms to be introduced below. Benefits of tapering the data have been widely reported in the literature. For example, tapers help to reduce leakage effects, especially when the spectral density contains high peaks. Also, the use of tapers leads to bias reduction, which is especially important when dealing with spatial data; namely, tapers can help to fight the so-called "edge effects" (see, e.g., [11, 14]).

Consider the tapered values

$$
\left\{h_{T}(t) X(t), t \in D_{T}\right\}
$$

where $h_{T}(t)=h(t / T), t=\left(t^{(1)}, \ldots, t^{(d)}\right) \in \mathbb{R}^{d}$, and the taper $h(t)$ factorizes as

$$
h(t)=\prod_{i=1}^{d} \tilde{h}\left(t^{(i)}\right), \quad t^{(i)} \in \mathbb{R},
$$

with $\tilde{h}(\cdot)$ satisfying

Assumption 1. $\tilde{h}(t), t \in \mathbb{R}$, is a positive even function of bounded variation with bounded support: $\tilde{h}(t)=0$ for $|t|>1$.

Denote

$$
\tilde{H}_{k, T}(\lambda)=\int \tilde{h}_{T}(t)^{k} e^{-i \lambda t} \nu(d t)
$$

and

$$
H_{k, T}(\lambda)=\int h_{T}(t)^{k} e^{-i(\lambda, t)} \nu(d t)=\prod_{i=1}^{d} \tilde{H}_{k, T}\left(\lambda^{(i)}\right) .
$$


These are the so-called spectral windows. The above integrals are one-dimensional and $d$-dimensional, respectively, with corresponding measure $\nu(\cdot)$ (for the discrete case we will deal actually with sums).

We next define the finite Fourier transform of tapered data $\left\{h_{T}(t) X(t), t \in D_{T}\right\}$ :

$$
d_{T}^{h}(\lambda)=\int h_{T}(t) X(t) e^{-i(\lambda, t)} \nu(d t), \quad \lambda \in \mathbb{S},
$$

the tapered periodograms of the second and the third orders:

$$
I_{2, T}^{h}(\lambda)=\frac{1}{(2 \pi)^{d} H_{2, T}(0)} d_{T}^{h}(\lambda) d_{T}^{h}(-\lambda)
$$

(provided that $H_{2, T}(0) \neq 0$ ),

$$
I_{3, T}^{h}\left(\lambda_{1}, \lambda_{2}\right)=\frac{1}{(2 \pi)^{2 d} H_{3, T}(0)} d_{T}^{h}\left(\lambda_{1}\right) d_{T}^{h}\left(\lambda_{2}\right) d_{T}^{h}\left(-\lambda_{1}-\lambda_{2}\right)
$$

(provided that $H_{3, T}(0) \neq 0$ ) and the tapered periodogram of $k$-th order:

$$
I_{k, T}^{h}\left(\lambda_{1}, \ldots, \lambda_{k-1}\right)=\frac{1}{(2 \pi)^{(k-1) d} H_{k, T}(0)} \prod_{i=1}^{k} d_{T}^{h}\left(\lambda_{i}\right), \quad \lambda_{i} \in \mathbb{S}
$$

(provided that $H_{k, T}(0) \neq 0$ ), where $\sum_{i=1}^{k} \lambda_{i}=0$, but no proper subset of $\lambda_{i}$ has sum 0 (that is, there is no other subset $\left\{\lambda_{i}, i \in \nu\right\}$, where $\nu=\left\{i_{1}, \ldots, i_{l}\right\} \subset\{1, \ldots, k\}$ and

$$
\sum_{i \in \nu} \lambda_{i}=0
$$

We will call these subsets submanifolds (see, e.g., [8, 7]).

The statistic (4) is a natural generalization of the second-order periodogram and can be considered as an estimator for the spectral density of $k$-th order $f_{k}\left(\lambda_{1}, \ldots, \lambda_{k-1}\right)$ at frequencies lying off submanifolds. To be more precise, a consistent estimator of $f_{k}\left(\lambda_{1}, \ldots, \lambda_{k-1}\right)$ can be produced as a weighted average of values (44) in the neighborhood of spatial frequencies of interest, but avoiding points on submanifolds (see, e.g., 8, 7, 22 ).

Substituting the periodogram $I_{k, T}^{h}(\lambda), \lambda \in \mathbb{S}^{k-1}, k=2,3, \ldots$, instead of the spectral density $f_{k}(\lambda)$ in the integral (2), we obtain the empirical spectral functional of $k$-th order

$$
J_{k, T}\left(\varphi_{k}\right)=\int_{S^{k-1}} \varphi_{k}(\lambda) I_{k, T}^{h}(\lambda) d \lambda .
$$

We will consider the question: can (5) work as a 'good' estimate for the spectral functional (2)?

Our main concern in the present paper is an evaluation of the bias of the estimators of spectral functionals and the conditions which guarantee an appropriate rate of convergence of a bias to zero, especially for spatial data $(d \geq 2)$ when the bias can be subject to edge effects.

Apparently, one of the first authors who addressed the problem of edge effects was Guyon [13]: considering the usual parametric Whittle estimates for lattice data, he found that such estimates had bias of order $N^{-1 / d}$ for fields observed on a rectangle

$$
P_{N}=\left\{1, \ldots, n_{1}\right\} \times \cdots \times\left\{1, \ldots, n_{d}\right\}
$$

in $\mathbb{Z}^{d}$, with $N$ being $\prod_{i=1}^{d} n_{i}$. Indeed, the number of boundary points increases with dimension, being of order $n^{d-1}$ for a cube of edge $n$. If we consider the estimate for the covariances $\gamma(k)$ by

$$
c_{N}=N^{-1} \sum_{\left\{t, t+k \in P_{N}\right\}} X(t) X(t+k),
$$


there is a bias due to boundary which is of order $O\left(N^{-1 / d}\right)$ or $O\left(n^{-1}\right)$ if $P_{N}$ is a cube of edge $n$. That is, for $d \geq 2$, the bias is of the same order or a higher order as the standard deviation of $c_{N}$ which is usually $O\left(N^{-1 / 2}\right)$. Analogously one obtains that the bias of the empirical spectral functional (5D), constructed with the usual untapered periodogram $(h(t) \equiv 1)$ behaves as $n^{-1}=N^{-1 / d}$. One possible solution to the described edge effect problem arising for spatial data is tapering the data at the edges of the observation domain.

We should mention that empirical spectral functionals (5) have a form of smoothing of the periodogram, which means that such an undesirable property of the periodogram as its inconsistency when considered as an estimate of the spectral density itself would not be troublesome when dealing with the spectral functional (5).

For $d=1, k=2$ and $d=1, k=3$ (as long as a zero mean is assumed for the processes considered) $J_{k, T}\left(\varphi_{k}\right)$ under appropriate conditions on $\varphi_{k}$ and $f_{k}$ indeed can serve as an estimator for $J_{k}\left(\varphi_{k}\right)$ as numerous studies confirm (actually most of these studies concentrated on the case $k=2$ and under the assumption of weak dependence).

Problems start to arise for $k=2,3$ but $d>1$, and particular attention should be paid now for maintaining a bias due to edge effects. A tool here would be an appropriate taper in dimensions $d=2,3$, as well as $d=1$. This has been shown in the literature for the cases $k=2,3$. We mention another approach to overcome bias due to edge effects which is to define the periodogram by means of unbiased estimators for moments/cumulants.

However more precaution is needed for higher orders $(k>3)$ as inconveniences appear due to the submanifolds (see the formula (9) below for the expectation of $J_{k, T}\left(\varphi_{k}\right)$ for $k>3$ ). To overcome this kind of difficulties, we introduce some corrections into the integrals (5) (see Section 2). We will also discuss another possibility to use (5) for the estimation of (2).

Finally, we should note that particular difficulties arise for the processes and fields with long-range dependence, as in this case the singularities of the spectral densities start to play a role. One way to resolve these difficulties is considering the trade-off between conditions of smoothness of the functions $\varphi_{k}$ and spectral densities $f_{k}$.

A common approach in the evaluation of bias of the estimators of spectral integrals of the second order is via asymptotic analysis of the following representation of the bias:

$$
\begin{aligned}
E J_{2, T}\left(\varphi_{2}\right)-J_{2}\left(\varphi_{2}\right) & =\iint \varphi_{2}(\lambda)\left(f_{2}(\lambda+u)-f_{2}(\lambda)\right) K_{2, T}(u) d u d \lambda \\
& =\iint f_{2}(\lambda)\left(\varphi_{2}(\lambda+u)-\varphi_{2}(\lambda)\right) K_{2, T}(u) d u d \lambda,
\end{aligned}
$$

using the smoothness properties of $\varphi_{2}$ and $f_{2}$ and the properties of the kernels $K_{2, T}$, which in the tapered case are of the form

$$
K_{2, T}=K_{2, T}^{h}(u)=\left((2 \pi)^{d} H_{2, T}(0)\right)^{-1}\left|H_{1, T}(u)\right|^{2} .
$$

Analogous representations can be exploited also for $J_{k, T}\left(\varphi_{k}\right)$ with $k>2$ as we will show below.

Although the cases $k=2,3$ have been studied in the literature, we prefer to consider these cases here as well in order to partly revise existing results and to present all the results in a unified manner. Our results concerning the case $k>3$ are stated in the next section, which are followed by discussions and conjectures on several directions for further research. To the best of our knowledge, the problem of bias due to edge effects for spectral estimates of higher orders has not been considered in the literature. The proof of the main result is given in Section 3 . 


\section{Results AND Discussions}

The expectation of the empirical spectral functional (5) is given by

$$
\begin{aligned}
& \mathrm{E} J_{k, T}\left(\varphi_{k}\right)=\frac{1}{(2 \pi)^{(k-1) d} H_{k, T}(0)} \int_{\mathbb{S}^{k-1}} \varphi_{k}(\lambda) \mathrm{E}\left\{\prod_{i=1}^{k} d_{T}^{h}\left(\lambda_{i}\right)\right\} \delta\left(\sum_{i=1}^{k} \lambda_{j}\right) d \lambda^{\prime} \\
& =\frac{1}{(2 \pi)^{(k-1) d} H_{k, T}(0)} \sum_{\substack{\nu=\left(\nu_{1}, \ldots, \nu_{p}\right) \\
\left|\nu_{i}\right|>1}} \int_{\mathbb{S}^{k-1}} \varphi_{k}(\lambda) \int_{\mathbb{S}^{k-p}} \prod_{i=1}^{p} f_{\left|\nu_{i}\right|}\left(\gamma_{j}, j \in \widetilde{\nu}_{i}\right) \\
& \times \prod_{j=1}^{k} H_{1, T}\left(\gamma_{j}-\lambda_{j}\right) \prod_{i=1}^{p} \delta\left(\sum_{j \in \nu_{i}} \gamma_{j}\right) d \gamma^{\prime} \delta\left(\sum_{i=1}^{k} \lambda_{j}\right) d \lambda^{\prime} \\
& =\frac{1}{(2 \pi)^{(k-1) d} H_{k, T}(0)} \int_{\mathbb{S}^{k-1}} \varphi_{k}(\lambda) \int_{\mathbb{S}^{k-1}} f_{k}\left(\gamma_{1}, \ldots, \gamma_{k-1}\right) \\
& \times \prod_{j=1}^{k} H_{1, T}\left(\gamma_{j}-\lambda_{j}\right) \delta\left(\sum_{j=1}^{k} \gamma_{j}\right) d \gamma^{\prime} \delta\left(\sum_{i=1}^{k} \lambda_{j}\right) d \lambda^{\prime} \\
& +\frac{1}{(2 \pi)^{(k-1) d} H_{k, T}(0)} \sum_{\substack{\nu=\left(\nu_{1}, \ldots, \nu_{p}\right) \\
p \geq 2,\left|\nu_{i}\right|>1}} \int_{\mathbb{S}^{k-1}} \varphi_{k}(\lambda) \int_{\mathbb{S}^{k-p}} \prod_{i=1}^{p} f_{\left|\nu_{i}\right|}\left(\gamma_{j}, j \in \widetilde{\nu}_{i}\right) \\
& \times \prod_{j=1}^{k} H_{1, T}\left(\gamma_{j}-\lambda_{j}\right) \prod_{i=1}^{p} \delta\left(\sum_{j \in \nu_{i}} \gamma_{j}\right) d \gamma^{\prime} \delta\left(\sum_{i=1}^{k} \lambda_{j}\right) d \lambda^{\prime} \\
& =I_{1}+I_{2} \text {, }
\end{aligned}
$$

where the summation in (77), (8) extends over all partitions $\nu=\left(\nu_{1}, \ldots, \nu_{p}\right)$ of the set $(1, \ldots, k)$. The above expression is obtained using the formula for cumulants of products of random variables (see [16]).

Here and in similar formulae below, we use the following notation: having a set of natural numbers $\nu$, we write $|\nu|$ to denote the number of elements in $\nu$, and $\widetilde{\nu}$ to denote the subset of $\nu$ which contains all elements of $\nu$ except the last one. In all partitions here and in what follows, $\left|\nu_{i}\right|>1$ for all $i$ (since we consider zero-mean fields). Integration in the inner integrals (see lines (7), (8) ) is understood with respect to the $(k-p) d$ dimensional vector $\gamma^{\prime}$, obtained from the $k d$-dimensional vector $\gamma=\left(\gamma_{1}, \ldots, \gamma_{k}\right)$ due to $p$ restrictions on the variables $\gamma_{j}, j=1, \ldots, k$, described by the Kronecker deltas $\delta(\cdot)$, and analogously for the integrals over the $\lambda$ 's. The same notation will be used in the similar integrals below.

The term $I_{2}$ does not appear if $k=2$ and $k=3$ (for zero-mean fields), which makes the asymptotic analysis of $\mathrm{E} J_{k, T}\left(\varphi_{k}\right)$ much simpler. Here we can apply standard arguments if we impose conditions of regularity on the spectral densities and weight functions $\varphi$, or, more generally, on their convolution. We state the results for the cases $k=2$ and $k=3$ in the next two theorems. The theorems provide conditions which assure the desirable rate of convergence of the bias of $J_{k, T}(\varphi)$ for $d=1,2,3$. We will need the following assumptions.

Assumption 2. The taper $\tilde{h}(t)$ is a Lipschitz-continuous function on $[-1,1]$.

Assumption 3. The function

$$
\varkappa_{2}^{h}(u)=\left|\int \tilde{h}(t) e^{-i t u} d t\right|^{2}
$$


satisfies

$$
\int|u|^{l} \varkappa_{2}^{h}(u)(u) d u<\infty, \quad l=1,2 .
$$

Theorem 2.1. Let the taper $\tilde{h}(t)$ satisfy Assumption 2 for discrete-parameter fields and Assumption 3 for continuous-parameter fields. Suppose further that one of the following conditions holds:

(i) $f_{2}$ is twice boundedly differentiable and $\varphi_{2} \in L_{1}(\mathbb{S})$,

(ii) $\varphi_{2}$ is twice boundedly differentiable,

(iii) the convolution $g_{2}(u)=\int f_{2}(\lambda) \varphi(\lambda+u) d \lambda$ is twice boundedly differentiable at zero.

Then, as $T \rightarrow \infty$,

$$
\mathrm{E} J_{2, T}\left(\varphi_{2}\right)-J_{2}\left(\varphi_{2}\right)=O\left(T^{-2}\right) .
$$

Assumption 4. The function $\varkappa_{3}^{h}(u)=\int \tilde{h}(t) e^{-i t u} d t \int \tilde{h}^{2}(t) e^{i t u} d t$ satisfies

$$
\int|u|^{l} \varkappa_{3}^{h}(u)(u) d u<\infty, \quad l=1,2 .
$$

Theorem 2.2. Let the taper $\tilde{h}(t)$ satisfy Assumption 2 for discrete-parameter fields and Assumption 4 for continuous-parameter fields. Suppose further that one of the following conditions holds:

(i) $f_{3}$ is twice boundedly differentiable and $\varphi_{3} \in L_{1}\left(\mathbb{R}^{2 d}\right)$,

(ii) $\varphi_{3}$ is twice boundedly differentiable,

(iii) the convolution $g_{3}\left(u_{1}, u_{2}\right)$ is twice boundedly differentiable at zero.

Then, as $T \rightarrow \infty$,

$$
\mathrm{E} J_{3, T}\left(\varphi_{3}\right)-J_{3}\left(\varphi_{3}\right)=O\left(T^{-2}\right) .
$$

Remark 2.1. 1. We can see that if the standard normalizing factor $T^{d / 2}$ is applied (under the conditions of Theorems 1 and 2), then the bias will be of order $T^{d / 2-2}$; that is, we can handle dimensions $d=1,2,3$ using the tapered periodogram in the estimators for $J_{2}(\varphi)$ and $J_{3}(\varphi)$.

2. When estimating the integrals of the spectrum and bispectrum, one has a possibility for a trade-off between the smoothness of a spectral density $f$ and that of the weight function $\varphi$ : as Theorems 1,2 show, one can relax the conditions on $f$ but imposing at the same time stronger conditions on $\varphi$. This allows us to treat the case of processes with long-range dependence.

For continuous-parameter fields, Theorems 1 and 2 were presented in [18; arguments for the proof in the discrete case are the same as those for the analysis of the term $I_{1}$ in the proof of Theorem 3 below (see also [11, 17] for an analysis of bias of spectral density estimators based on tapered data).

For the case $k>3$, the term $I_{2}$ in the expression (9) for $\mathrm{E} J_{k, T}\left(\varphi_{k}\right)$ creates inconveniences. One possible way to overcome these difficulties is to exclude from consideration the points lying on submanifolds, as prescribed in the definition of the periodogram of order $k>3$ (see formula (44)), and to consider the empirical spectral functional

$$
J_{k, T}^{*}\left(\varphi_{k}\right)=J_{k, T, \varepsilon}^{*}\left(\varphi_{k}\right)=\int_{\mathbb{S}_{\varepsilon}^{k-1}} \varphi_{k}(\lambda) I_{k, T}^{h}(\lambda) d \lambda
$$

as an estimator for

$$
J_{k}^{*}\left(\varphi_{k}\right)=J_{k, \varepsilon}^{*}\left(\varphi_{k}\right)=\int_{\mathbb{S}_{\varepsilon}^{k-1}} \varphi_{k}(\lambda) f_{k}(\lambda) d \lambda
$$


for all $\varepsilon>0$, where the integration is taken over $\mathbb{S}^{k-1}$, but avoiding the frequencies on and neighboring to the submanifolds

$$
\left\{\sum_{i \in \nu} \lambda_{i}=0\right\}, \quad \text { where } \nu=\left\{i_{1}, \ldots, i_{l}\right\} \subset\{1, \ldots, k\} .
$$

More precisely,

$$
\mathbb{S}_{\varepsilon}^{k-1}=\mathbb{S}^{k-1} \backslash\left\{\lambda:\left|\sum_{i \in \nu} \lambda_{i}\right|<\varepsilon \text { for all } \nu \subset\{1, \ldots, k\}\right\},
$$

where $|y|=\max _{1 \leq i \leq d}\left|y^{(i)}\right|$.

We will need one more assumption.

Assumption 5. The function

$$
\varkappa_{k}^{h}(u)=\int \tilde{h}(t) e^{-i t u} d t \int \tilde{h}^{k-1}(t) e^{i t u} d t
$$

satisfies

$$
\int|u|^{l} \varkappa_{k}^{h}(u)(u) d u<\infty, \quad l=1,2 .
$$

Theorem 2.3. Let the taper $\tilde{h}(t)$ satisfy Assumption 2 for discrete-parameter fields and Assumption 5 for continuous-parameter fields. Suppose further that the spectral density of the $k$-th order $f_{k}(\lambda)$ is twice boundedly differentiable, $\varphi_{k} \in L_{1}\left(\mathbb{S}^{k-1}\right)$, and the spectral densities $f_{l}(\lambda) \in L_{k-2}\left(\mathbb{S}^{l-1}\right)$ for $l=2, \ldots, k-2$. Then as $T \rightarrow \infty$,

$$
\mathrm{E} J_{k, T}^{*}\left(\varphi_{k}\right)-J_{k}^{*}\left(\varphi_{k}\right)=O\left(T^{-2}\right) \text {. }
$$

For the discrete-parameter random fields we have an immediate corollary. We can consider the estimator $J_{k, T}^{*}\left(\varphi_{k}\right)=J_{k, T, \varepsilon}^{*}\left(\varphi_{k}\right)$ with $\varepsilon=1 / T$. Let us denote this estimator as $J_{k, T}^{* *}\left(\varphi_{k}\right)$.

Theorem 2.4. For the case of discrete-parameter fields, let the taper $\tilde{h}(t)$ satisfy Assumption 2. Suppose further that the spectral density of the $k$-th order $f_{k}(\lambda)$ is twice boundedly differentiable, $\varphi_{k} \in L_{1}\left(\mathbb{S}^{k-1}\right)$, and the spectral densities $f_{l}(\lambda) \in L_{k-2}\left(\mathbb{S}^{l-1}\right)$ for $l=2, \ldots, k-2$.

Then as $T \rightarrow \infty$,

$$
\mathrm{E} J_{k, T}^{* *}\left(\varphi_{k}\right)-J_{k}\left(\varphi_{k}\right)=O\left(T^{-2}\right)+O\left(T^{-d}\right) .
$$

In light of Theorems 3 and 4, the results of 1 can be reformulated under the conditions which now become operational. For continuous-parameter fields, we cannot state (with the technique we used for the proofs) the analogous result in general, but only under some additional conditions on $\varphi_{k}$ and $f_{k}$ prescribing their specific behavior in the neighborhood of submanifolds. Since such conditions would be somewhat artificial, we will not pursue the result further.

Remark 2.2. 1. As we have already noted above, the bias of order $O\left(T^{-2}\right)$ allows us to handle the dimensions $d=1,2,3$ and to study the estimators further (their consistency, asymptotic normality): the standard normalizing factor $T^{d / 2}$ can be used. Some authors considered the problem of nonparametric estimation of the higher-order cumulant spectra of stationary random fields by smoothing (nontapered) periodograms; the bias of such estimators was evaluated to behave as $n^{-1}$ if the domain of observation is a cube of edge $n$ (see, e.g., 22]). In such a case, asymptotic normality results can be stated only for the spectral estimate with its mean value subtracted, as was done in 22 . 
2. In the discrete-time case, the domain over which the field is observed has been traditionally taken to be $D_{T}=[1, T]^{d}$. Our results remain valid for such a domain as well; we just need to adjust the assumptions on a taper $\tilde{h}(t)$. Namely, Assumption 1 must be modified as follows: $\tilde{h}(t)$ is a positive function of bounded variation with support on $[0,1]$ and $h(0)=0, h(1-v)=h(v)$ for $0 \leq v \leq \frac{1}{2}$.

3. An example of a taper $\tilde{h}(t)$ satisfying the assumptions introduced in the discreteparameter case is

$$
\tilde{h}(t)=\frac{1}{2}(1+\cos (4 \pi t)), \quad t \in[-1,1] .
$$

This is a modification of the well-known cosine bell (or the Tukey-Hanning taper)

$$
\tilde{h}(t)=\frac{1}{2}(1-\cos (2 \pi t)), \quad t \in[0,1],
$$

suitable for the domain $D_{T}=[1, T]^{d}$.

For the continuous-parameter case, we can consider the taper

$$
\tilde{h}(t)=1-|t| \text { for }|t| \leq 1 .
$$

The corresponding spectral window $\tilde{H}_{1}(\lambda)$ is of the form

$$
\tilde{H}_{1}(\lambda)=(\sin (\lambda / 2))^{2} /(\lambda / 2)^{2} .
$$

Another example is the taper

$$
\tilde{h}(t)=\left(1-|t|^{2}\right)^{\alpha} \quad \text { for }|t| \leq 1 \text { and } \alpha>1 / 2,
$$

where $|t|=(t, t)^{1 / 2}$, with the spectral window of the form

$$
\tilde{H}_{1}(\lambda)=(2 \pi)^{-1 / 2} 2^{\alpha} \Gamma(\alpha+1) J_{1 / 2+\alpha}(|\lambda|)|\lambda|^{-(1 / 2+\alpha)} .
$$

4. We have considered in this paper the case of the taper $h(t), t \in \mathbb{R}^{d}$, which factorizes:

$$
h(t)=\prod_{i=1}^{d} \tilde{h}\left(t^{(i)}\right), \quad t^{(i)} \in \mathbb{R},
$$

since this assumption makes all derivations much simpler and allows us to directly exploit known results for the one-dimensional case. However we feel that this assumption is too restrictive, and it would be interesting to study the general situation, when a taper does not factorize. An example of tapers of interest is

$$
h(t)=\left(1-|t|^{2}\right)^{\alpha} \quad \text { for }|t| \leq 1
$$

with the spectral window of the form

$$
H_{1}(\lambda)=(2 \pi)^{-d / 2} 2^{\alpha} \Gamma(\alpha+1) J_{d / 2+\alpha}(|\lambda|)|\lambda|^{-(d / 2+\alpha)} .
$$

5. The proof of Theorem 3 relies heavily on the technique developed in [9, 10, which is based on suitable upper bounds for spectral windows $H_{k, T}(\lambda)$. These bounds are given by the specific function $L_{T}(\lambda)$ (see the proof in the next section), whose convolution properties help to simplify the analysis and allow us to impose rather weak conditions on spectral densities (such as integrability conditions). However, with this approach, tapers are treated in such a unified manner that we just use the assumption that they are of bounded variation (in addition to some other general assumptions) and we do not look at more specific features of tapering functions. We believe that, by considering particular cases of tapering functions, more refined results can be obtained. In particular, as noted above, we cannot state the analog of Theorem 4 for continuous-parameter fields. This limitation is caused by the technique we use for the proofs, but can be overcome, as we expect, by means of other methods. 
6. The approach considered in the paper for construction of spectral estimators of higher orders consists of excluding the contribution due to the points on submanifolds (following [8, 7, 22]). Another approach is to consider as an estimator for (2) the empirical functional (5) from which we subtract some other empirical functionals to compensate for the integrals appearing in the term $I_{2}$ in formula (16). This kind of estimator was considered in [19] for the fourth-order spectral functional which appears in the asymptotic variance for the quasi-maximum likelihood estimator in the non-Gaussian case. General estimators of this kind for higher-order spectral functionals, constructed recursively, were studied in [15].

We will address the questions raised in the above remarks in our future research.

\section{Proof of Theorem 3}

Similar to the formula for $\mathrm{E} J_{k, T}\left(\varphi_{k}\right)$ and using the same notation we have

$$
\begin{aligned}
\mathrm{E} J_{k, T}^{*}\left(\varphi_{k}\right)=\int_{\mathbb{S}_{\varepsilon}^{k-1}} \varphi_{k}(\lambda) \frac{1}{(2 \pi)^{(k-1) d} H_{k, T}(0)} \mathrm{E}\left\{\prod_{i=1}^{k} d_{T}^{h}\left(\lambda_{i}\right)\right\} \delta\left(\sum_{i=1}^{k} \lambda_{j}\right) d \lambda^{\prime} \\
=\frac{1}{(2 \pi)^{(k-1) d} H_{k, T}(0)} \sum_{\substack{\nu=\left(\nu_{1}, \ldots, \nu_{p}\right) \\
\left|\nu_{i}\right|>1}} \int_{\mathbb{S}_{\varepsilon}^{k-1}} \varphi_{k}(\lambda) \int_{\mathbb{S}^{k-p}} \prod_{i=1}^{p} f_{\left|\nu_{i}\right|}\left(\gamma_{j}, j \in \widetilde{\nu}_{i}\right) \\
\times \prod_{j=1}^{k} H_{1, T}\left(\gamma_{j}-\lambda_{j}\right) \prod_{i=1}^{p} \delta\left(\sum_{j \in \nu_{i}} \gamma_{j}\right) d \gamma^{\prime} \delta\left(\sum_{i=1}^{k} \lambda_{j}\right) d \lambda^{\prime} \\
=\frac{1}{(2 \pi)^{(k-1) d} H_{k, T}(0)} \int_{\mathbb{S}_{\varepsilon}^{k-1}}^{\varphi_{k}(\lambda) \int_{\mathbb{S}^{k-1}} f_{k}\left(\gamma_{1}, \ldots, \gamma_{k-1}\right)} \\
\times \prod_{j=1}^{k} H_{1, T}\left(\gamma_{j}-\lambda_{j}\right) \delta\left(\sum_{j=1}^{k} \gamma_{j}\right) d \gamma^{\prime} \delta\left(\sum_{i=1}^{k} \lambda_{j}\right) d \lambda^{\prime} \\
\quad+\frac{1}{(2 \pi)^{(k-1) d} H_{k, T}(0)} \sum_{\nu=\left(\nu_{1}, \ldots, \nu_{p}\right)}^{p} \int_{\mathbb{S}_{\varepsilon}^{k-1}} \varphi_{k}(\lambda) \int_{\mathbb{S}^{k-p}} \prod_{i=1}^{k} f_{\left|\nu_{i}\right|}\left(\gamma_{j}, j \in \widetilde{\nu}_{i}\right) \\
\times \prod_{j=1}^{k} H_{1, T}\left(\gamma_{j}-\lambda_{j}\right) \prod_{i=1}^{p} \delta\left(\sum_{j \in \nu_{i}} \gamma_{j}\right) d \gamma^{\prime} \delta\left(\sum_{i=1}^{k} \lambda_{j}\right) d \lambda^{\prime} \\
=I_{1}+I_{2} . \quad
\end{aligned}
$$

To analyze the term $I_{1}$ we consider the continuous and discrete cases separately. Consider firstly the continuous case $\left(\mathbb{S}=\mathbb{R}^{d}\right)$. We can write

$$
\begin{aligned}
I_{1}=\frac{1}{(2 \pi)^{(k-1) d} H_{k, T}(0)} \int_{\mathbb{S}_{\varepsilon}^{k-1}} \varphi_{k}(\lambda) \int_{\mathbb{S}^{k-1}} f_{k}\left(\lambda_{1}+u_{1}, \ldots, \lambda_{k-1}+u_{k-1}\right) \\
\quad \times \prod_{j=1}^{k} H_{1, T}\left(u_{j}\right) \delta\left(\sum_{j=1}^{k} u_{j}\right) d u^{\prime} \delta\left(\sum_{i=1}^{k} \lambda_{j}\right) d \lambda^{\prime},
\end{aligned}
$$

where

$$
H_{k, T}(0)=T^{d}\left(\tilde{H}_{k}(0)\right)^{d}, \quad \prod_{j=1}^{k} H_{1, T}\left(u_{j}\right)=T^{k d} \prod_{j=1}^{k} \prod_{i=1}^{d} \tilde{H}_{1}\left(T u_{j}^{(i)}\right)
$$


and $\tilde{H}_{k}(u)=\int \tilde{h}(t)^{k} e^{-i t u} d t$. Changing the variables $T u_{j}^{(i)}=v_{j}^{(i)}$, we obtain

$$
\begin{aligned}
I_{1}=\frac{1}{(2 \pi)^{(k-1) d}\left(\tilde{H}_{k}(0)\right)^{d}} \int_{\mathbb{S}_{\varepsilon}^{k-1}} \varphi_{k}(\lambda) & \int_{\mathbb{S}^{k-1}} f_{k}\left(\lambda_{1}+\frac{v_{1}}{T}, \ldots, \lambda_{k-1}+\frac{v_{k-1}}{T}\right) \\
& \times \prod_{j=1}^{k} \prod_{i=1}^{d} \tilde{H}_{1}\left(v_{j}^{(i)}\right) \delta\left(\sum_{j=1}^{k} v_{j}\right) d v^{\prime} \delta\left(\sum_{i=1}^{k} \lambda_{j}\right) d \lambda^{\prime} .
\end{aligned}
$$

By the assumptions of the theorem, $f_{k}$ is twice boundedly differentiable; therefore, using Taylor's theorem, we can write

$$
f_{k}\left(\lambda_{1}+\frac{v_{1}}{T}, \ldots, \lambda_{k-1}+\frac{v_{k-1}}{T}\right)=f_{k}(\lambda)+\mathrm{const} \cdot T^{-1} \sum_{j=1}^{k-1} \sum_{i=1}^{d} v_{j}^{(i)}+O\left(T^{-2}\right) \sum_{j=1}^{k-1} \sum_{i=1}^{d}\left|v_{j}^{(i)}\right|^{2}
$$

(uniformly in $\lambda$ in the $O$-term). Correspondingly, for $I_{1}$ we obtain

$$
I_{1}=\int_{\mathbb{S}_{\varepsilon}^{k-1}} \varphi_{k}(\lambda) f_{k}(\lambda) d \lambda^{\prime}+R_{1}+R_{2} .
$$

We now evaluate $R_{1}$ and $R_{2}$. The error term $R_{1}$ is of the form

$$
\begin{aligned}
R_{1}=\mathrm{const} \cdot \int_{\mathbb{S}_{\varepsilon}^{k-1}} & \varphi_{k}(\lambda) \frac{1}{(2 \pi)^{(k-1) d}\left(\tilde{H}_{k}(0)\right)^{d}} \\
& \times \frac{1}{T} \sum_{j=1}^{k-1} \sum_{i=1}^{d} \int_{\mathbb{S}^{k-1}} v_{j}^{(i)} \prod_{j=1}^{k} \prod_{i=1}^{d} \tilde{H}_{1}\left(v_{j}^{(i)}\right) \delta\left(\sum_{j=1}^{k} v_{j}\right) d v^{\prime} \delta\left(\sum_{i=1}^{k} \lambda_{j}\right) d \lambda^{\prime} .
\end{aligned}
$$

This reduces to the sum of terms of the form

$$
\text { const } \cdot \frac{1}{T} \int_{\mathbb{S}_{\varepsilon}^{k-1}} \varphi_{k}(\lambda) \int_{\mathbb{R}} v_{j}^{(i)} \tilde{H}_{1}\left(v_{j}^{(i)}\right) \tilde{H}_{k-1}\left(-v_{j}^{(i)}\right) d v_{j}^{(i)} \delta\left(\sum_{i=1}^{k} \lambda_{j}\right) d \lambda^{\prime},
$$

each of which is equal to zero since $\tilde{H}_{1}\left(v_{j}^{(i)}\right) \tilde{H}_{k-1}\left(-v_{j}^{(i)}\right)$ is an even function.

Analogously, $R_{2}$ reduces to the sum of terms of the form

$$
\text { const } \cdot \int_{\mathbb{S}_{\varepsilon}^{k-1}} \varphi_{k}(\lambda) \int_{\mathbb{R}}\left|v_{j}^{(i)}\right|^{2} \tilde{H}_{1}\left(v_{j}^{(i)}\right) \tilde{H}_{k-1}\left(-v_{j}^{(i)}\right) d v_{j}^{(i)} \delta\left(\sum_{i=1}^{k} \lambda_{j}\right) d \lambda^{\prime},
$$

supplied with the multiplier $O\left(T^{-2}\right)$. In view of Assumption 5 and the integrability of $\varphi_{k}$, the integrals (17) are bounded; therefore, we obtain the following asymptotics for $I_{1}$ :

$$
I_{1}=\int_{\mathbb{S}_{\varepsilon}^{k-1}} \varphi_{k}(\lambda) f_{k}(\lambda) d \lambda^{\prime}+O\left(T^{-2}\right) .
$$

Consider now the discrete case $\left(\mathbb{S}=(-\pi, \pi]^{d}\right)$. We get

$$
\begin{aligned}
I_{1}=\frac{1}{(2 \pi)^{(k-1) d} H_{k, T}(0)} \int_{\mathbb{S}_{\varepsilon}^{k-1}} \varphi_{k}(\lambda) \int_{\mathbb{S}^{k-1}} f_{k}\left(\lambda_{1}+u_{1}, \ldots, \lambda_{k-1}+u_{k-1}\right) \\
\quad \times \prod_{j=1}^{k} H_{1, T}\left(u_{j}\right) \delta\left(\sum_{j=1}^{k} u_{j}\right) d \gamma^{\prime} \delta\left(\sum_{i=1}^{k} \lambda_{j}\right) d \lambda^{\prime},
\end{aligned}
$$

where $\prod_{j=1}^{k} H_{1, T}\left(u_{j}\right)=\prod_{j=1}^{k} \prod_{i=1}^{d} \tilde{H}_{1, T}\left(u_{j}^{(i)}\right)$ and $H_{k, T}(0) \sim T^{d}\left(\tilde{H}_{k}(0)\right)^{d}$ as $T \rightarrow \infty$. 
By Taylor's theorem and the assumption that $f_{k}(\lambda)$ is twice boundedly differentiable on $\mathbb{S}^{k-1}$,

$$
\left|f_{k}\left(\lambda_{1}+u_{1}, \ldots, \lambda_{k-1}+u_{k-1}\right)-f_{k}(\lambda)-\sum_{j=1}^{k-1} \sum_{i=1}^{d} u_{j}^{(i)} \frac{\partial f_{k}(\lambda)}{\partial \lambda_{j}^{(i)}}\right| \leq \text { const } \cdot \sum_{j=1}^{k-1} \sum_{i=1}^{d}\left|u_{j}^{(i)}\right|^{2} .
$$

We denote

$$
\alpha_{T}=I_{1}-\int_{\mathbb{S}_{\varepsilon}^{k-1}} \varphi_{k}(\lambda) f_{k}(\lambda) d \lambda^{\prime}
$$

and consider the expression

$$
\begin{aligned}
\frac{1}{(2 \pi)^{(k-1) d} H_{k, T}(0)} \sum_{j=1}^{k-1} \sum_{i=1}^{d} \int_{\mathbb{S}_{\varepsilon}^{k-1}} \varphi_{k}(\lambda) \frac{\partial f_{k}(\lambda)}{\partial \lambda_{j}^{(i)}} \int_{\mathbb{S}^{k-1}} u_{j}^{(i)} \\
\times \prod_{j=1}^{k} \prod_{i=1}^{d} \tilde{H}_{1, T}\left(u_{j}^{(i)}\right) \delta\left(\sum_{j=1}^{k} u_{j}\right) d u^{\prime} \delta\left(\sum_{i=1}^{k} \lambda_{j}\right) d \lambda^{\prime} .
\end{aligned}
$$

The inner integrals in (18) reduce to the expressions

$$
\left\{(2 \pi)^{(k-1)} \tilde{H}_{k, T}(0)\right\}^{d-1}(2 \pi)^{(k-2)} \int_{-\pi}^{\pi} u_{j}^{(i)} \tilde{H}_{1, T}\left(u_{j}^{(i)}\right) \tilde{H}_{k-1, T}\left(-u_{j}^{(i)}\right) d u_{j}^{(i)}
$$

and the last integral is equal to zero since $\tilde{H}_{1, T}\left(u_{j}^{(i)}\right) \tilde{H}_{k-1, T}\left(-u_{j}^{(i)}\right)$ is an even function. We conclude that (18) is equal to zero and

$$
\begin{aligned}
\left|\alpha_{T}\right| \leq \text { const } & \cdot \sum_{j=1}^{k-1} \sum_{i=1}^{d} \int_{\mathbb{S}_{\varepsilon}^{k-1}}\left|\varphi_{k}(\lambda)\right| \\
\times\left[\frac{1}{(2 \pi)^{(k-1) d} H_{k, T}(0)} \int_{\mathbb{S}^{k-1}}\left|u_{j}^{(i)}\right|^{2} \prod_{j=1}^{k} \prod_{i=1}^{d}\left|\tilde{H}_{1, T}\left(u_{j}^{(i)}\right)\right| \delta\left(\sum_{j=1}^{k} u_{j}\right) d u^{\prime}\right] & \times \delta\left(\sum_{i=1}^{k} \lambda_{j}\right) d \lambda^{\prime} .
\end{aligned}
$$

Now we take into account the following facts:

(i) $H_{k, T}(0) \sim T^{d}\left(\tilde{H}_{k}(0)\right)^{d}$ as $T \rightarrow \infty$,

(ii) for the taper $\tilde{h}(t)$ of bounded variation,

$$
\left|\tilde{H}_{1, T}(u)\right| \leq \mathrm{const} \cdot \tilde{L}_{T}(u)
$$

where the function $\tilde{L}_{T}(u)$ is the periodic extension (with period $2 \pi$ ) of the function $\tilde{L}_{T}^{*}(u)$ defined as follows: $\tilde{L}_{T}^{*}(u)=T$ for $|u| \leq 1 / T$, and $\tilde{L}_{T}^{*}(u)=1 /|u|$ for $1 / T<|u| \leq \pi$

(iii) $\int_{-\pi}^{\pi} \tilde{L}_{T}(u) \tilde{L}_{T}(v-u) d u \leq$ const $\cdot L_{T}(v)$

(see [9, Lemma 2]). An application of (ii) and (iii) implies that

$$
\int_{(-\pi, \pi]^{k-1}} \prod_{j=1}^{k}\left|\tilde{H}_{1, T}\left(u_{j}\right)\right| \delta\left(\sum_{j=1}^{k} u_{j}\right) d u_{1} \cdots d u_{k-1} \leq \mathrm{const} \cdot L_{T}(0) \leq \mathrm{const} \cdot T .
$$

This allows us to conclude that the expression in square brackets in (19) is bounded by

$$
\text { const } \cdot \frac{1}{T} \int_{-\pi}^{\pi}|u|^{2}\left|\tilde{H}_{1, T}(u) \tilde{H}_{k-1, T}(-u)\right| d u \text {. }
$$


Then the inequality

$$
\begin{aligned}
& \int_{-\pi}^{\pi}|u|^{2}\left|\tilde{H}_{1, T}(u) \tilde{H}_{k-1, T}(-u)\right| d u \\
& \quad \leq\left(\int_{-\pi}^{\pi}|u|^{2}\left|\tilde{H}_{1, T}(u)\right|^{2} d u\right)^{1 / 2}\left(\int_{-\pi}^{\pi}|u|^{2}\left|\tilde{H}_{k-1, T}(-u)\right|^{2} d u\right)^{1 / 2}
\end{aligned}
$$

holds. Using the fact that a taper function $\tilde{h}(t)$ is Lipschitz-continuous, we have the estimate

$$
\int_{-\pi}^{\pi}\left|\tilde{H}_{k, T}(u)\right|^{2} d u \leq \frac{1}{T}
$$

(see, e.g., [17). Therefore, each term on the left hand side of (19) is bounded by const $\cdot T^{-2}$, which gives the asymptotics for $I_{1}$ the same as in the continuous case:

$$
I_{1}=\int_{\mathbb{S}_{\varepsilon}^{k-1}} \varphi_{k}(\lambda) f_{k}(\lambda) d \lambda^{\prime}+O\left(T^{-2}\right) .
$$

Returning to the expression (16) for $\mathrm{E} J_{k, T}^{*}\left(\varphi_{k}\right)$, we see that we still have to evaluate $I_{2}$. Here we will use the same reasoning in both the continuous and discrete cases $\left(\mathbb{S}=\mathbb{R}^{d}\right.$ and $\left.\mathbb{S}=(-\pi, \pi]^{d}\right)$ using a technique found in [9, 10. Under the assumption that $\tilde{h}(t)$ is of bounded variation, we can write the following bounds for the spectral windows:

$$
\left|H_{1, T}(u)\right| \leq \operatorname{const} \cdot L_{T}(u),
$$

where $L_{T}(u)=\prod_{i=1}^{d} \tilde{L}_{T}\left(u^{(i)}\right)$ and the function $\tilde{L}_{T}(u)$ for the discrete case has just been defined (see (ii) above) and in the continuous case this function is defined as follows: $\tilde{L}_{T}(u)=T$ for $|u| \leq 1 / T$, and $\tilde{L}_{T}(u)=1 /|u|$ for $|u|>1 / T$.

We will use the convolution properties of the functions $\tilde{L}_{T}(u)$ : for $p>1$,

$$
\int_{\tilde{S}} \tilde{L}_{T}^{p}(x+u) \tilde{L}_{T}^{p}(y-u) d u \leq \mathrm{const} \cdot T^{p-1} \tilde{L}_{T}^{p}(x+y),
$$

where $\tilde{\mathbb{S}}=\mathbb{R}$ or $(-\pi, \pi]$ for the continuous and discrete cases respectively (see [9] and [12] for the discrete and continuous cases respectively).

We can write

$$
\begin{aligned}
&\left|I_{2}\right| \leq \sum_{\substack{\nu=\left(\nu_{1}, \ldots, \nu_{p}\right) \\
p \geq 2,\left|\nu_{i}\right|>1}} \int_{\mathbb{S}_{\varepsilon}^{k-1}}\left|\varphi_{k}(\lambda)\right| \frac{1}{(2 \pi)^{(k-1) d} H_{k, T}(0)} \\
& \times \int_{\mathbb{S}^{k-p}} \prod_{i=1}^{p}\left|f_{\left|\nu_{i}\right|}\left(\gamma_{j}, j \in \widetilde{\nu}_{i}\right)\right| \prod_{j=1}^{k}\left|H_{1, T}\left(\gamma_{j}-\lambda_{j}\right)\right| \\
& \times \prod_{i=1}^{p} \delta\left(\sum_{j \in \nu_{i}} \gamma_{j}\right) d \gamma^{\prime} \delta\left(\sum_{i=1}^{k} \lambda_{j}\right) d \lambda^{\prime} .
\end{aligned}
$$

Suppose we can apply the Hölder inequality to bound the inner integral in (20) by the following expression:

$$
\begin{aligned}
\text { const }\left\{\int_{\mathbb{S}^{k-p}} \prod_{i=1}^{p}\left|f_{\left|\nu_{i}\right|}\left(\gamma_{j}, j \in \widetilde{\nu}_{i}\right)\right|^{r} \prod_{i=1}^{p} \delta\left(\sum_{j \in \nu_{i}} \gamma_{j}\right) d \gamma^{\prime}\right\}^{1 / r} \\
\times\left\{\int_{\mathbb{S}^{k-p}} \prod_{j=1}^{k} L_{T}^{q}\left(\gamma_{j}-\lambda_{j}\right) \prod_{i=1}^{p} \delta\left(\sum_{j \in \nu_{i}} \gamma_{j}\right) d \gamma^{\prime}\right\}^{1 / q}
\end{aligned}
$$


where $r, q>1, r^{-1}+q^{-1}=1$. The term in the second brackets in (21) is equal to

$$
\begin{aligned}
& \prod_{l=1}^{p} \int_{\mathbb{S}\left|\nu_{l}\right|-1} \prod_{j \in \nu_{l}} L_{T}^{q}\left(\gamma_{j}-\lambda_{j}\right) \prod_{i=1}^{p} \delta\left(\sum_{j \in \nu_{l}} \gamma_{j}\right) d \gamma^{\prime} \\
& \quad=\prod_{l=1}^{p} \prod_{i=1}^{d} \int_{\tilde{\mathbb{S}}\left|\nu_{l}\right|-1} \prod_{j \in \nu_{l}} \tilde{L}_{T}^{q}\left(\gamma_{j}^{(i)}-\lambda_{j}^{(i)}\right) \prod_{i=1}^{p} \delta\left(\sum_{j \in \nu_{l}} \gamma_{j}^{(i)}\right) d \gamma^{\prime}
\end{aligned}
$$

which, using the convolution properties of the function $\tilde{L}_{T}(u)$, can be bounded by

$$
\prod_{l=1}^{p} \prod_{i=1}^{d}\left\{T^{(q-1)\left(\left|\nu_{l}\right|-1\right)} \tilde{L}_{T}^{q}\left(\sum_{j \in \nu_{l}} \lambda_{j}^{(i)}\right)\right\}=T^{(q-1)(k-p) d} \prod_{l=1}^{p} \prod_{i=1}^{d} \tilde{L}_{T}^{q}\left(\sum_{j \in \nu_{l}} \lambda_{j}^{(i)}\right) .
$$

Therefore we obtain the following bound for the terms of the sum (20):

$$
\text { const } \cdot T^{(q-1)(k-p) d / q} \int_{\mathbb{S}_{\varepsilon}^{k-1}}\left|\varphi_{k}(\lambda)\right| \prod_{l=1}^{p} \prod_{i=1}^{d} \tilde{L}_{T}\left(\sum_{j \in \nu_{l}} \lambda_{j}^{(i)}\right) \delta\left(\sum_{i=1}^{k} \lambda_{j}\right) d \lambda^{\prime} .
$$

In view of specific domains of integration and a specific form of the arguments of the functions $\tilde{L}_{T}$, for sufficiently large $T$ these functions can be bounded by $1 / T$, and with the choice

$$
q=\frac{k-2}{k-3},
$$

the above expression will be of order $O\left(T^{-2}\right)$ as $T \rightarrow \infty$. Therefore, under the assumption that $f_{l}(\lambda) \in L_{k-2}\left(\mathbb{S}^{l-1}\right)$ for $l=2, \ldots, k-2$ we have

$$
I_{2}=O\left(T^{-2}\right) \quad \text { as } T \rightarrow \infty
$$

which completes the proof.

\section{BIBLIOGRAPHY}

1. V. V. Anh, N. N. Leonenko, and L. M. Sakhno, Quasilikelihood-based higher-order spectral estimation of random processes and fields with possible long-range dependence, J. Appl. Probab. 41A (2004), 35-54. MR2057564 (2005j:62169)

2. V. V. Anh, N. N. Leonenko, and L. M. Sakhno, Minimum contrast estimation of random processes based on information of second and third orders, J. Statist. Plann. Inference 137 (2007), 1302-1331. MR2301481 (2008h:62218)

3. V. V. Anh, N. N. Leonenko, and L. M. Sakhno, Statistical inference using higher-order information, J. Multivariate Anal. 98 (2007), no. 4, 706-742. MR.2322125 (2009a:62345)

4. R. Bentkus, Cumulants of estimates of the spectrum of a stationary sequence, Liet. Mat. Rink. 16 (1976), 37-61. (Russian) MR0431571 (55:4568)

5. R. Bentkus and R. Rutkauskas, On the asymptotics of the first two moments of second order spectral estimators, Litovsk. Mat. Sb. 13 (1973), 29-45. (Russian) MR0319334 (47:7878)

6. R. Bentkus, R. Rutkauskas, and Ju. Sušinskas, The average of the estimates of the spectrum of a homogeneous field, Liet. Mat. Rink. 14 (1974), 67-74. (Russian) MR0386193 (52:7051)

7. D. R. Brillinger, The frequency analysis of relations between stationary spatial series, Proceedings of the Twelfth Biennial Seminar of the Canadian Mathematical Congress, Montreal, 1970, pp. 39-81. MR0273763(42:8640)

8. D. R. Brillinger and M. Rosenblatt, Asymptotic theory of estimates of $k$-th order spectra, Spectral Anal. Time Ser. (B. Harris, ed.), Wiley, New York, 1967. MR.0211566 (35:2444)

9. R. Dahlhaus, Spectral analysis with tapered data, J. Time Ser. Anal. 4 (1983), 163-175. MR732895 (85c:62246)

10. R. Dahlhaus, A functional limit theorem for tapered empirical spectral functions, Stoch. Process. Appl. 19 (1985), 135-149. MR780726 (86i:60089)

11. R. Dahlhaus and H. Künsch, Edge effects and efficient parameter estimation for stationary random fields, Biometrika 74 (1987), 877-882, 39-81. MR.919857(89g:62146) 
12. M. Eichler, Empirical spectral processes and their applications to stationary point processes, Ann. Appl. Probab. 5 (1995), no. 4, 1161-1176. MR.1384370 (97k:60062)

13. X. Guyon, Parameter estimation for a stationary process on a d-dimensional lattice, Biometrika 69 (1982), 95-105. MR655674 (83j:62128)

14. X. Guyon, Random Fields on a Network: Modelling, Statistics and Applications, Springer, New York, 1995. MR1344683 (96m:60119)

15. D. M. Keenan, Limiting behavior of functionals of higher-order sample cumulant spectra, Ann. Stat. 15 (1987), no. 1, 134-151. MR885728 (88k:62176)

16. V. V. Leonov and A. N. Shiryaev, On a method of calculation of semi-invariants, Theor. Probab. Appl. 4 (1959), 319-329. MR0123345 (23:A673)

17. P. M. Robinson, Nonparametric spectrum estimation for spatial data, J. Statist. Plann. Inference 137 (2007), no. 3, 1024-1034. MR2301732 (2008m:62157)

18. L. Sakhno, Bias control in the estimation of spectral functionals, Theory Stoch. Process. 1329 (2007), no. 1-2, 225-233. MR2343825 (2008m:62087)

19. M. Taniguchi, On estimation of the integrals of the fourth order cumulant spectral density, Biometrika 69 (1982), 117-122. MR655676 (83g:62144)

20. M. Taniguchi, Minimum contrast estimation for spectral densities of stationary processes, J. R. Stat. Soc., Ser. B 49 (1987), 315-325. MR928940(89c:62163)

21. J. Yuan and T. Subba Rao, Spectral estimation for random fields with applications to Markov modelling and texture classification, Markov Random Fields: Theory and Applications (R. Chellappa and A. K. Jain, eds.), Academic Press, Boston, 1993, pp. 179-209. MR1214376 (93j:68224)

22. J. Yuan and T. Subba Rao, Higher order spectral estimation for random fields, Multidimensional Syst. Signal Process. 4 (1993), no. 1, 7-22. MR.1202189 (93k:62198)

School of Mathematical Sciences, Queensland University of Technology, GPO Box 2434, Brisbane QLD 4001, Australia

E-mail address: v.anh@qut.edu.au

School of Mathematics, Cardiff University, Senghennydd Road, Cardiff CF24 4YH, United KINGDOM

E-mail address: LeonenkoN@Cardiff.ac.uk

Department of Mechanics and Mathematics, Kyiv National Taras Shevchenko University, Volodymyrska 64, Kyiv 01033, Ukraine

E-mail address: Ims@univ.kiev.ua

Received 12/MAR/2009

Originally published in English 